\title{
Lifetime enhancement of scroll rings by spatiotemporal fluctuations
}

\author{
V. Pérez-Muñuzuri, ${ }^{1, *}$ F. Sagués, ${ }^{2}$ and J. M. Sancho ${ }^{3}$ \\ ${ }^{1}$ Group of Nonlinear Physics, Faculty of Physics, University of Santiago de Compostela, 15706 Santiago de Compostela, Spain \\ ${ }^{2}$ Departament de Química Física, Universitat de Barcelona, Avenida Diagonal 647, 08028 Barcelona, Spain \\ ${ }^{3}$ Departament d'Estructura i Constituents de la Matèria, Universitat de Barcelona, Avenida Diagonal 647, 08028 Barcelona, Spain
}

(Received 11 January 2000)

\begin{abstract}
The dynamics of three-dimensional scroll rings with spatiotemporal random excitability is investigated numerically using the FitzHugh-Nagumo model. Depending on the correlation time and length scales of the fluctuations, the lifetime of the ring filament is enlarged and a resonance effect between the time scale of the scroll ring and the time correlation of the noise is observed. Numerical results are interpreted in terms of a simplified stochastic model derived from the kinematical equations for three-dimensional excitable waves.
\end{abstract}

PACS number(s): 82.40.Bj, 41.20.Jb, 47.54. $+\mathrm{r}$

\section{INTRODUCTION}

Excitable media describe systems ranging from the biological to the chemical. These systems usually show pattern formation under the form of spiral waves in two-dimensional (2D) media or more complex structures such as scroll waves in 3D media $[1,2]$. While in the first case the tip of the spiral rotates describing a trajectory that can be circular or even more complex, in the 3D case the scroll waves rotate around a one-dimensional singularity known as the filament. In the simplest case, this filament is a straight line but it may also be curved along a circle giving rise to the scroll rings, or even twisted resulting in a twisted scroll wave.

The dynamics of the vortex filaments in reaction-diffusion systems has attracted a great deal of attention $[3,4]$ in connection with sudden heart fibrillation, where it is believed that scroll ring vortices may play a crucial role $[5,6]$. In heterogeneous systems, such as the Belousov-Zhabotinsky (BZ) chemical reaction and the cardiac tissue $[7,8]$, these $3 \mathrm{D}$ waves usually propagate in the presence of inhomogeneities. Usually, two types of heterogeneities have been studied in excitable media; passive ones, nonreactive and noninteracting with the medium, and active ones which affect the wave dynamics. With respect to the first category, the presence of localized inhomogeneities or quenched noise in the medium gives rise to anchoring of vortices in 2D [9] and 3D [10] media, wave front distortion [11], or even wave front breaking. Effects of spatiotemporal correlated noises are examples of the second class of heterogeneities and have also been studied within the framework of dynamical control of waves in excitable media: drift, resonance, etc. [12]. Moreover, it has been shown that spatiotemporal noise is able to sustain pattern formation in the subexcitable regime [13-15].

On the other hand, both in homogeneous and heterogeneous excitable conditions, scroll rings are one of the most unstable structures that develop in these media $[16,17]$. They usually contract (at a rate proportional to the diffusion coefficient and inversely proportional to the radius of the ring) or expand and finally disappear, accompanied by a drift of the

\footnotetext{
*URL: http://fmares.usc.es; Electronic address: vicente@fmmeteo.usc.es
}

ring along its symmetry axis. Shrinkage proceeds until a minimum radius is reached at which the ring abruptly collapses. Recent work by Vinson et al. $[18,19]$ with parameter gradients allows us to some extent to control the lifetime of these structures. Previous numerical and theoretical calculations by Mikhailov $[20,21]$ showed that a periodic modulation of a parameter controlling the filament curvature could lead to controlling the rate of expansion or contraction of the wave.

In this paper, we are interested in the control of scroll rings with spatiotemporal correlated noise, since it accounts for the two effects described above; on the one hand, the correlation length of the noise disturbs the wave propagation distorting the front, and on the other hand, the time correlation affects the dynamics of the medium. To address this situation, a model of the FitzHugh-Nagumo type, incorporating parametric noise, is proposed in Sec. II and numerical results for the dynamics of the ring filament are presented in Sec. III. Theoretical analysis, Sec. IV, is based on a minimal stochastic model with multiplicative noise, originally obtained within a kinematic description of scroll rings.

\section{THE MODEL}

Numerical simulations have been performed with the modified FitzHugh-Nagumo model [19,22,23],

$$
\begin{gathered}
\frac{\partial E}{\partial t}=f(E)-g+D \nabla^{2} E, \\
\frac{\partial g}{\partial t}=\frac{E-g}{\epsilon(E)},
\end{gathered}
$$

where $E$ stands for the excitation of the medium, $g$ is the recovery variable, and $f(E)$ and $\epsilon(E)$ are given by the piecewise functions,

$$
f(E)= \begin{cases}-c_{1} E, & E<E_{1} \\ c_{2}(E-a), & E_{1} \leqslant E \leqslant E_{2} \\ -c_{3}(E-1), & E>E_{2},\end{cases}
$$




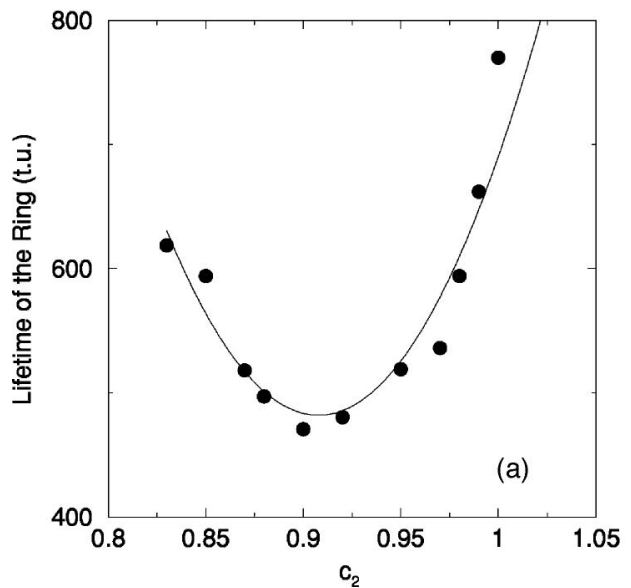

$$
\epsilon(E)= \begin{cases}\epsilon_{1}, & E<B_{1} \\ \epsilon_{2}, & B_{1} \leqslant E \leqslant B_{2} \\ \epsilon_{3}, & E>B_{2} .\end{cases}
$$

The parameter values for Eqs. (1)-(3) are defined as in Ref. [19]: $\quad c_{1}=4.0, \quad c_{3}=15.0, \quad E_{1}=0.018, \quad \epsilon_{1}=\epsilon_{3}=0.5, \quad \epsilon_{2}$ $=16.66, B_{1}=0.01, B_{2}=0.95$, and $D=1$. The parameters $E_{2}$ and $a$ are determined by continuity of function $f(E) ; E_{2}$ $=\left[\left(c_{1}+c_{2}\right) E_{1}+c_{3}\right] /\left(c_{3}+c_{2}\right), a=E_{1}\left(c_{1}+c_{2}\right) / c_{2}$.

Initial conditions correspond to a scroll ring generated from a spherical wave in the center of the medium (by stimulating a $4 \times 4 \times 4$ pixel cube at $E=0.5$ ), and then resetting half of the medium $(E=0, g=0) 20$ t.u. (time unit) later so the initial radius of the ring is approximately 23 s.u. (space unit). With this set of parameters, initial conditions, and without any further perturbation, a scroll ring is generated which finally collapses while it drifts along its vertical axis.

The set of equations (1)-(3) has been integrated using a Euler method with a time step $\Delta t=0.04$ t.u. and the Laplacian operator in Eq. (1) discretized using a seven-point centered difference scheme with a space step $\Delta=0.75$ s.u. Besides, zero flux boundary conditions were considered for the $E$ variable. The mesh size of the grid was $100 \times 100$

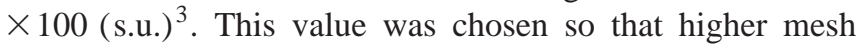
sizes do not affect qualitatively the obtained results.

The spatial structure of the filament has been resolved by following a procedure with mixed characteristics of the two described in Refs. [7,19]. The filament is defined as the intersection line between two surfaces $E(\vec{r}, t)=E_{\text {iso }}$, where $E_{\text {iso }}$ is some arbitrary threshold value used to distinguish excitable and inhibited regions, and $\partial_{t} E(\vec{r}, t)=0$. This is equivalent [7] to solving, for each lattice plane $z=k \Delta$ $=$ const, the set of equations

$$
\begin{aligned}
& (1-\bar{x})(1-\bar{y}) E_{i, j, k}^{n}+\bar{x}(1-\bar{y}) E_{i+1, j, k}^{n}+\bar{x} \bar{y} E_{i+1, j+1, k}^{n} \\
& \quad+(1-\bar{x}) \bar{y} E_{i, j+1, k}^{n}=E_{\mathrm{iso}}, \\
& (1-\bar{x})(1-\bar{y}) E_{i, j, k}^{n+1}+\bar{x}(1-\bar{y}) E_{i+1, j, k}^{n+1}+\bar{x} \bar{y} E_{i+1, j+1, k}^{n+1} \\
& \quad+(1-\bar{x}) \bar{y} E_{i, j+1, k}^{n+1}=E_{\mathrm{iso}},
\end{aligned}
$$

where $\bar{x}=(x-i \Delta) / \Delta, \bar{y}=(y-j \Delta) / \Delta$, and $E^{n}, E^{n+1}$ are consecutive iteration values of $E$ resulting from the integration
FIG. 1. Free-noise lifetime of the scroll ring (a) and rotation velocity of the scroll wave (b) as a function of the parameter $c_{2}$ from the FitzHugh-Nagumo model. The solid lines represent a fit to the numerical data with a polynomial function. of Eq. (1). Equations (4) and (5) have solution if $0 \leqslant \bar{x} \leqslant 1$ and $0 \leqslant \bar{y} \leqslant 1$. This solution represents the intersection of the filament with the square with corners $(i, j),(i+1, j),(i, j$ $+1)$ and $(i+1, j+1)(k=$ const $)$. Points of intersections with all squares are then determined by a similar procedure. On the other hand, since the filament could be folded, at each time step the set of Eqs. (4) and (5) is also solved for lattice planes of $y=j \Delta=$ const, which is equivalent to exchanging $z$ and $k$ by $y$ and $j$ above. Then, the final position of the filament $(\bar{x}, \bar{y}, \bar{z})$ within the cube $(i, j, k)$ is calculated as a linear interpolation between the two obtained values. At the next time step this algorithm is repeated but only within the neighborhood of the previous positions.

Once the filament has been located, we fit those points to a plane as it is described by Vinson and Pertsov [19], which allows us to determine the mean radius of the filament $\rho$, as well as the $z$ value of the "center of mass" of the filament for the case of rings.

The lifetime of the scroll ring is then defined as the interval of time $T$ that the ring radius is greater than zero during wave contraction or the $z$ coordinate of the center of mass of the filament is within the grid of simulation. To calibrate the medium and to get some insight into where to introduce external fluctuations, the lifetime of the scroll ring and the rotation velocity of the scroll wave have been calculated within the range of $c_{2}$ values used [Figs. 1(a) and 1(b)]. The lifetime is observed to follow a second-order power dependence with $c_{2}$, while the rotation velocity exhibits a linear dependence as $c_{2}$ increases. This last property induces us to consider this parameter as a good candidate to introduce fluctuations.

Spatiotemporal noise was added to the system through the parameter $c_{2}$ (generally varying between 0.80 and 1.02 for excitable conditions $), c_{2}(\vec{r}, t)=c_{2}^{0}+\xi(\vec{r}, t) \quad\left(c_{2}^{0}=0.88\right)$, and $\vec{r}=(x, y, z)$. After discretization, the random term $\xi_{i j k}(t)$ is defined in larger cubic domains, or cells with centered coordinates $(i, j, k)$, of size $\ell^{3}$, where $\ell>\Delta$. It is prescribed as a spatiotemporal Gaussian noise of zero mean and correlation [24],

$$
\left\langle\xi_{i j k}(t) \xi_{l m n}\left(t^{\prime}\right)\right\rangle=\sigma^{2} e^{-\left|t-t^{\prime}\right| / \tau} \delta_{i l} \delta_{j m} \delta_{k n}
$$

where $\tau$ denotes its correlation time and $\sigma^{2}$ is the noise second moment. The parameter $\ell$ fixes the characteristic 


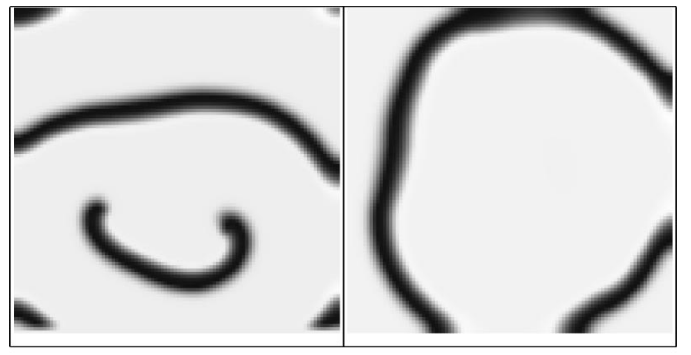

FIG. 2. Vertical ( $x z$ plane, $y=50)$ (left image) and horizontal ( $x y$ plane, $z=50$ ) (right image) profiles of the ring wave. Time $=440$ t.u. Parameters of the numerical simulation: $\sigma=0.05$ and $\ell$ $=20$ s.u.

length of the inhomogeneous fluctuating excitability. In the limit $\tau \rightarrow 0$ the white-noise limit $\xi_{w}(t)$ is recovered if $\sigma^{2} \tau$ is finite. For $\tau \rightarrow \infty$ the frozen or static Gaussian distributed noise is obtained. Numerical simulations have been carried out by keeping constant the noise dispersion $\sigma^{2}$ while varying the time correlation $\tau$ and the noise cell size $\ell$.

\section{NUMERICAL RESULTS}

Numerical simulations indicate that wave fronts are distorted as a consequence of the spatiotemporal correlated noise. Figure 2 shows two profiles of the ring wave 440 t.u. after noise was switched on. The perfect mirror symmetry of the two counter-rotating spiral waves in the homogeneous case was broken as a consequence of the fluctuating disorder in the medium. Nevertheless, the ring filament, determined by the procedure described above, still keeps a circular geometry as it is shown in Fig. 3.

Figure 4 shows the temporal evolution of the ring radius $\rho(t)$ for three realizations of noise with the same correlation length $\ell$ but different correlation time $\tau$. For comparison, the lifetime of the ring free of noise is also indicated as a vertical dashed line. Note that the extinction time of the ring free of noise $T=497$ t.u. is always smaller than the cases when fluctuations are present, and that for a particular value of the correlation time $\tau$, the lifetime is very large. The ring was found to collapse for a finite, but still small value of $\rho$ $\neq 0$. This can be easily understood if we reduce the dimen-

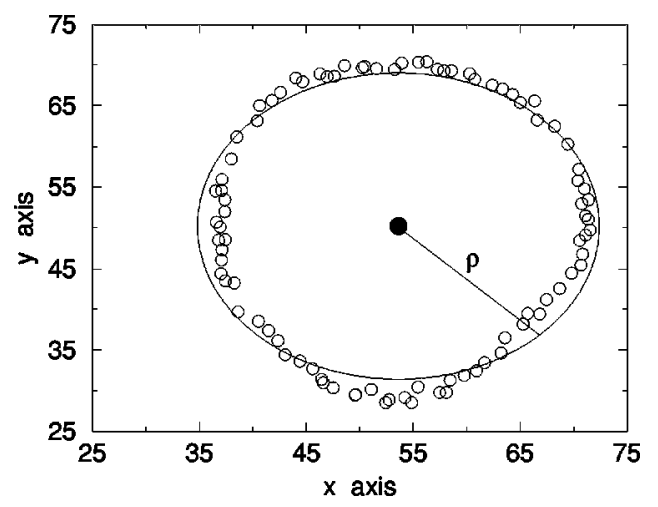

FIG. 3. Filament of the ring for $t=400$ t.u. The line shows the fitting of the filament positions to a circle in order to determine its radius $\rho=18.8$ s.u. Note that although the presence of noise is clear due to the data scatters around the circle, still a nice round behavior is preserved. Parameters as in Fig. 2.

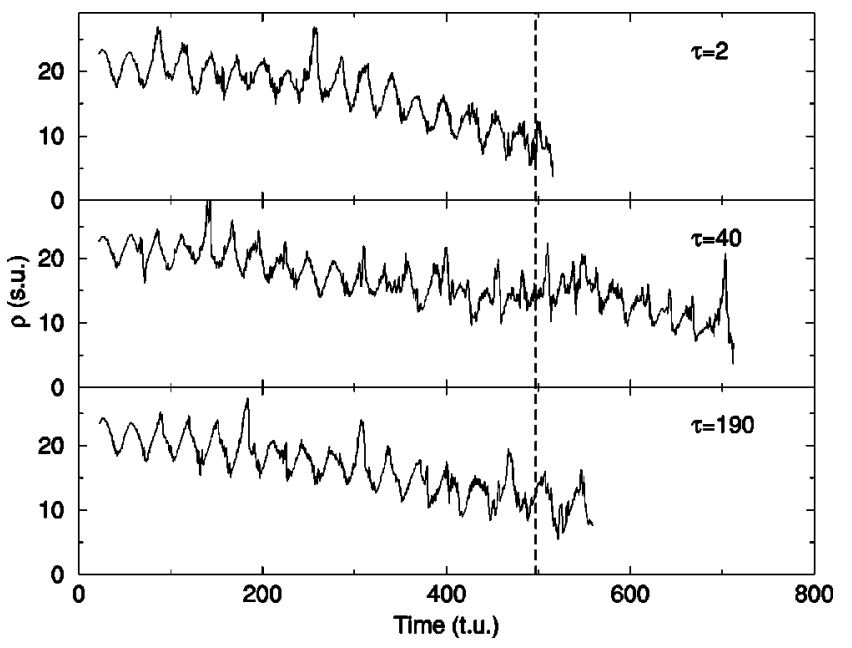

FIG. 4. Temporal evolution of $\rho(t)$ for three different realizations of the colored noise. The vertical dashed line indicates the lifetime of the scroll wave in the free noise case $T=497$ t.u. Note the temporal oscillations corresponding to the rotation period of the scroll wave around the filament and approximately equal to 29 t.u. Due to the presence of noise, these oscillations are not perfectly regular. Parameters as in Fig. 2.

sion of the problem to an axial plane of the scroll ring where two counter-rotating 2D spiral waves approach each other. Then, both spirals will annihilate each other when the relative distance between spiral tips is smaller than the core diameter [25].

A more quantitative study of the effect of the spatiotemporal correlated noise on the mean lifetime of scroll rings is shown in Fig. 5 for different values of the correlation length $\ell$. The most remarkable effect is the occurrence of a maximum lifetime of the ring for some intermediate value of the time correlation $\tau$. This occurs for all values of $\ell$ and seems to be the signature of a resonant behavior between the time scale of the ring wave (associated to the rotation period around the filament) and the noise correlation time. In addition, this maximun appears shifted to smaller values of $\tau$ when the correlation length $\ell$ increases. From the analysis of our numerical results we can conjecture that the limiting position of this maximum will appear at $\approx 1 / w_{0}=4.5$ t.u., where $w_{0}$ is the rotation frequency of the scroll wave around the filament, and would be achieved for $\ell$ equal to a very large system size $L$ relative to the initial radius of the scroll ring. Note also that the highest lifetimes are obtained again in this limit (when the noise is only time-correlated), while the resonance peaks are narrower. In any case, in all our simulations, the effect of a correlated noise was to induce the longer ring's lifetimes compared to the case without noise $c_{2}=c_{2}^{0}$, but always the filament finally contracts and disappears.

Independently of the correlation length $\ell$, the two limit cases of $\tau$, namely $\tau \rightarrow 0$ and $\tau \rightarrow \infty$, can be intuitively explained. For $\tau \rightarrow 0$ and finite $\sigma^{2}$, the white Gaussian noise limit with zero noise intensity is recovered. The control parameter $c_{2}$ fluctuates so fast compared with the time scales of the ring that, on average, the wave filament behaves as in the free noise situation, $c_{2}=\left\langle c_{2}\right\rangle=c_{2}^{0}$, while for $\tau \rightarrow \infty$ the noise becomes static and the filament tends to smoothly fold and become distorted [11] (mostly keeping a planar shape so a 

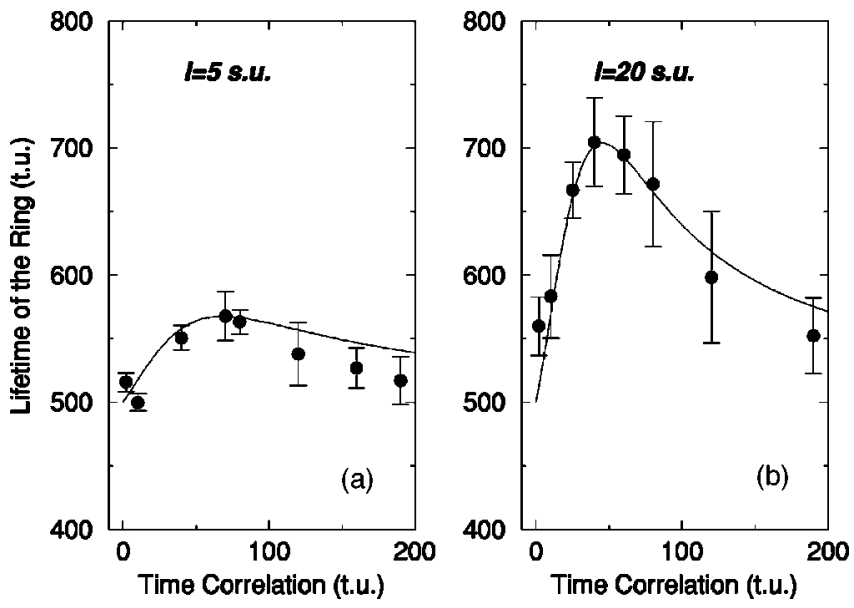

radius $\rho$ can still be easily defined) but it does not interfere in the contraction process.

\section{ANALYTICAL STUDY}

Scroll rings in three-dimensional excitable media have axial symmetry, in such a way that if we cut the 3D media with a plane containing the symmetry axis, two symmetrical counter-rotating spiral waves develop. In this case, the kinematical theory introduced by Mikhailov [20,21] can be readily applied under conditions of low excitability and the quasistationary approach. Although our numerical experiments fall beyond that limit, such a description enables us to capture, at least qualitatively, what we believe are the essential features of the effect of structured excitability fluctuations on 3D scroll rings. The equations that describe the dynamics of the scroll wave filament (which is seen in the axial plane as a single point) are

$$
\begin{gathered}
\dot{\rho}=-D / \rho-\left(V_{0}-D k_{0}\right) \sin \alpha_{0}-\widetilde{G} \cos \alpha_{0}, \\
\dot{z}=\left(V_{0}-D k_{0}\right) \cos \alpha_{0}-\widetilde{G} \sin \alpha_{0}, \\
\dot{\alpha}_{0}=\omega_{0}+\widetilde{G} k_{0}, \\
\dot{k}_{0}=\omega_{0} \widetilde{G} / D,
\end{gathered}
$$

where $V_{0}$ is the rotation velocity of the scroll wave, $k_{0}$ its curvature at the filament position, $\alpha_{0}$ is the angle between the tangent to the curve at the filament and the $\rho$ axis, $\omega_{0}$ $=\zeta \sqrt{D V_{0}} k_{0}^{3 / 2}(\zeta=0.685)$ is the rotation frequency of the scroll wave around the filament, and its tangential growth velocity $\widetilde{G}$ is given by

$$
\widetilde{G}=\left(G_{0}-\gamma_{1} k_{0}\right)+\rho^{-1}\left[\gamma_{1} \sin \alpha_{0}+\left(\gamma_{2}-D\right) \cos \alpha_{0}\right],
$$

where $\gamma_{1}$ and $\gamma_{2}$ are two positive coefficients that control the sprouting velocity around the filament in a $3 \mathrm{D}$ media. In general, it can be shown [20] that for a system with a single diffusive species [see Eq. (1)], $\gamma_{1}, \gamma_{2}$, and $D$ do not have to be equal to each other.

Thus we start from the set of equations (7)-(11) where for simplicity we make the assumption of a constant angular velocity $\alpha_{0}=\omega_{0} t$. We neglect also the curvature corrections

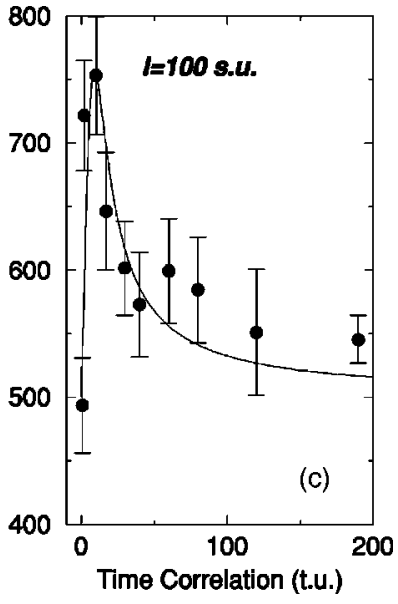

FIG. 5. Dependence of the scroll wave lifetime on the noise time correlation $\tau$ for three different values of the noise pixel size $\ell$ : (a) $\ell=5$ s.u., $d_{5}=0.14, b_{5}$ $=0.067 ;$ (b) $\ell=20, d_{20}=0.24$, $b_{20}=0.1$; and (c) $\ell=L=100, d_{L}$ $=1, b_{L}=0.5$. Error bars correspond to a statistical error for ten different realizations of noise. Theoretical results (24) are included for comparison. Numerical parameters as in Fig. 2 and theoretical common parameters: $\sigma_{L}^{2}$ $=0.48, x_{0}=400$ s.u., $a=0.8$.

to the normal propagation velocity, i.e., $V_{0}-D k_{0} \approx V_{0}$, and in fact we assume (see below) no temporal evolution at all for the variable $k_{0}$. Furthermore, since the temporal evolution of the pair of basic dynamic variables, $\rho(t)$ and $z(t)$, is apparently quite similar in our simulations, we restrict ourselves to the analysis of the dynamics of the variable $\rho(t)$, the radius of the filament. According to the approximations just quoted, the equation for $\rho(t)$ reads

$$
\dot{\rho}=-D / \rho-V_{0} \sin \left(\omega_{0} t\right)-\widetilde{G} \cos \left(\omega_{0} t\right)
$$

which is easily converted into the form

$$
\dot{\rho} \rho=-D-V_{0} \rho \sin \left(\omega_{0} t\right)-\widetilde{G} \rho \cos \left(\omega_{0} t\right) .
$$

We invoke now the additional equation (11) for the tangential growth velocity $\widetilde{G}$, which we use as

$$
\begin{aligned}
\widetilde{G} \rho \cos \left(\omega_{0} t\right)= & \left(G_{0}-\gamma_{1} k_{0}\right) \rho \cos \left(\omega_{0} t\right) \\
& +\gamma_{1} \sin \left(\omega_{0} t\right) \cos \left(\omega_{0} t\right)+\left(\gamma_{2}-D\right) \cos ^{2}\left(\omega_{0} t\right) .
\end{aligned}
$$

Using trigonometric relations, and substituting Eq. (14) into Eq. (13) above, we get

$$
\begin{aligned}
\dot{\rho} \rho= & -1 / 2 a-\left[V_{0} \sin \left(\omega_{0} t\right)+\left(G_{0}-\gamma_{1} k_{0}\right) \cos \left(\omega_{0} t\right)\right] \rho \\
& -1 / 2\left[\gamma_{1} \sin \left(2 \omega_{0} t\right)+\left(\gamma_{2}-D\right) \cos \left(2 \omega_{0} t\right)\right],
\end{aligned}
$$

where $a=\gamma_{2}+D$. We introduce now a new dynamical variable $\Pi(t) \equiv \rho^{2}(t)$, so that

$$
\begin{aligned}
\dot{\Pi}(t)= & -a-\left[\gamma_{1} \sin \left(2 \omega_{0} t\right)+\left(\gamma_{2}-D\right) \cos \left(2 \omega_{0} t\right)\right] \\
& -2\left[V_{0} \sin \left(\omega_{0} t\right)+\left(G_{0}-\gamma_{1} k_{0}\right) \cos \left(\omega_{0} t\right)\right] \Pi^{1 / 2}
\end{aligned}
$$

At this point, and according to what was mentioned in Sec. II concerning the relation between the parameter $c_{2}$ and the rotation velocity of the scroll wave, we choose to introduce fluctuations only through the kinematic parameter $V_{0}$, i.e., we replace $V_{0}$ by $\bar{V}_{0}+\chi(t)$. The statistical properties of this pure temporal noise $\chi(t)$ have to be obtained from those of the original spatiotemporal correlated noise (6). This is a very difficult and nonsystematic task, and hence the noise 
characteristics of $\chi(t)$ has to be somewhat conjectured from intuitive arguments on the coupling of the temporal and length scales of the dynamical disorder in the excitability with those of the scroll ring. Furthermore, we suppose that the contribution $\left(G_{0}-\gamma_{1} k_{0}\right)$ to the tangential growth velocity is negligible (this means that we are neglecting the systematic, i.e., non- $\rho$-dependent, contribution to the tangential growth velocity). In the framework of $2 \mathrm{D}$ spiral waves this assumption is merely considering rigid rotation of the spiral tip. With these hypotheses, we simplify the last equation into the form

$$
\begin{aligned}
\text { П }(t)= & -a-\gamma_{1} \sin \left(2 \omega_{0} t\right)-\left(\gamma_{2}-D\right) \cos \left(2 \omega_{0} t\right) \\
& -2\left[\bar{V}_{0}+\chi(t)\right] \sin \left(\omega_{0} t\right) \Pi^{1 / 2} .
\end{aligned}
$$

Just to finish, we discard from this equation the oscillatory terms not responsible for the systematic dynamics of the shrinking filament, but at the same time we keep the minimum contribution from the fluctuating environment,

$$
\Pi(t)=-a+\phi(t) \sin \left(\omega_{0} t\right) \Pi^{1 / 2},
$$

where $\phi=2 \chi$. This equation constitutes the minimum starting model but in this form it is still analytically intractable and further assumptions on the noise properties have to be taken. First, one can assume that $\phi$ is a Gaussian colored noise of the same type used in the simulations (OrnsteinUhlenbech process) of zero mean and correlation,

$$
\left\langle\phi(t) \phi\left(t^{\prime}\right)\right\rangle=\sigma_{\ell}^{2} e^{-\left|t-t^{\prime}\right| / \tau /},
$$

where $\sigma_{\ell}^{2}$ and $\tau_{\ell}$ should be proportional to the parameters $\sigma^{2}$ and $\tau$, respectively, used in the simulations, with multiplicative constants depending on $\ell$. A final redefinition of the fluctuating term allows us to express Eq. (18),

$$
\dot{\Pi}(t)=-a+\eta(t) \Pi^{1 / 2},
$$

where $\eta(t)=\phi(t) \sin \left(\omega_{0} t\right)$. Since we have seen in the numerical results that the lifetime of the scroll wave is much larger than the correlation time of the noise, one can approximate the noise in Eq. (20) by an effective stationary Gaussian white noise, $\left\langle\eta(t) \eta\left(t^{\prime}\right)\right\rangle=2 \epsilon \delta\left(t-t^{\prime}\right)$, with a renormalized intensity $\epsilon$ given by

$$
\epsilon=\frac{1}{2} \int_{0}^{\infty} \cos \left(\omega_{0} s\right)\langle\phi(s) \phi(0)\rangle d s=\frac{\sigma_{\ell}^{2}}{2}\left(\frac{\tau_{\ell}}{1+\omega_{0}^{2} \tau_{\ell}^{2}}\right) .
$$

Let us stress at this point that Eq. (20) in spite of the crudeness of its derivation contains the basic physical ingredients necessary to interpret our numerical results. In fact, notice that the ring dynamics retains its genuine deterministic component (rate of shrinking inversely proportional to its radius) supplemented with a multiplicative (state-dependent) noise, this last feature anticipating a pronounced effect of fluctuations on the scroll ring lifetime. In addition, within this formulation, the problem of ring collapse is reproduced by the stochastic dynamics of a positive random variable drifted towards an absorbing boundary placed at its zero value. Thus, we can evaluate the mean lifetime of the scroll ring by calculating the mean first passage time, $\left\langle T\left(x_{0}\right)\right\rangle$, of the variable $\Pi(t)$ to get for the first time the value $\Pi=0$ from an initial value $\Pi=x_{0}=\rho^{2}(0)$. Needless to say this also corresponds to the lifetime of the original variable $\rho(t)$. Whithin our Markovian approximation (20) and (21), $\left\langle T\left(x_{0}\right)\right\rangle$ obeys the differential equation [26]

$$
\left[\epsilon x_{0} \frac{\partial^{2}}{\partial x_{0}^{2}}-\left(a-\frac{\epsilon}{2}\right) \frac{\partial}{\partial x_{0}}\right]\left\langle T\left(x_{0}\right)\right\rangle=-1,
$$

with the boundary condition $\langle T(0)\rangle=0$. A particular solution of Eq. (22) which retains the dominant (quasideterministic) contribution gives

$$
\frac{\partial\left\langle T\left(x_{0}\right)\right\rangle}{\partial x_{0}}=\left(1-\frac{\epsilon}{a-\epsilon / 2} \frac{\partial}{\partial x_{0}}\right)^{-1} \frac{1}{a-\epsilon / 2}=\frac{1}{a-\epsilon / 2}
$$

Integrating now between 0 and $x_{0}$, we get that the mean lifetime of the variable $\Pi$ has a simple expression in terms of the effective noise intensity,

$$
\left\langle T\left(x_{0}\right)\right\rangle=\frac{x_{0}}{a-\epsilon / 2} .
$$

This is a remarkably simple analytical expression containing all the functional dependences and fluctuating effects through the only parameter $\epsilon\left(\sigma_{\ell}^{2}, \tau_{\ell}, \omega_{0}\right)$. We thus can conclude from it that $\left\langle T\left(x_{0}\right)\right\rangle$ is indeed larger than the deterministic exit time $T_{0}=x_{0} / a$ and has a maximun with a height controlled by $\sigma_{\ell}^{2}$ at a position given by $\tau_{\ell}=\omega_{0}^{-1}$.

Let us now compare this formal theoretical prediction with numerical simulations of the extended system. First we will consider the case of a spatiotemporal noise with a correlation length of the order of the system size, $L=100$. The excitability fluctuations are then homogeneous all over the spatial domain occupied by the scroll ring, with a temporal dependence parametrized by the correlation time $\tau_{L}=b_{L} \tau$. Actually, $b_{L} \neq 1$ due to the finite size of the system. From the parameter values $x_{0}, \omega_{0}$, and $\tau$ used in the numerical simulations and fitting the parameters $\sigma_{L}^{2}, b_{L}$, and $a$, we can compare the analytical result (24) with the numerical data shown in Fig. 5(c), with very good agreement.

Now we have to discuss the other situations when the noise has a finite correlation length $\ell$. Since the scroll ring is an extended structure under the influence of local values of the noise, one can conjecture that the effective noise acting on the radius is a sort of spatial average of the noise applied on the whole filament. This amounts to a reduction of the effective intensity with respect to the $L$ case, $\sigma_{\ell}^{2}=d_{\ell} \sigma_{L}^{2}$ $<\sigma_{L}^{2}$

Moreover, the scroll ring in its shrinking dynamics sweeps over a 3D grid of uncorrelated noise pixels resulting in an effective correlation time $\tau_{\ell}, \ell$-dependent, and smaller than the original one: $\tau_{\ell}=b_{\ell} \tau<\tau$. Adjusting the pair of parameters, $d_{\ell}$ and $b_{\ell}$ for the cases $\ell=5$ and $\ell$ $=20$, we present in Figs. 5(a) and 5(b), the analytical results compared with numerical simulation data. 


\section{CONCLUSIONS}

We have evidenced numerically, with a FitzHughNagumo and a very simplified stochastic model, the resonance behavior between the time scales of a 3D scroll ring in an excitable medium when it is forced with a spatiotemporal correlated noise. When increasing the correlation length of the noise $\ell$, the amplitude of the resonant peak increases and it appears at smaller values of $\tau$, while the peak becomes narrower. Finally, for $\ell$ of the order of the system size, the resonant peak occurs at the value of the rotation period.

Since 3D scroll rings are usually unstable, and they slowly shrink at a rate inversely proportional to the ring ra- dius to finally collapse, as a future work it will be interesting to study the role of correlated noises for stabilizing this process. Previous work [20,21] in this direction with periodic forcing suggests this possibility.

\section{ACKNOWLEDGMENTS}

We wish to thank Irene Sendiña-Nadal for fruitful discussions. This work was supported by DGES under Research Grant Nos. PB96-0241, PB96-1001, and PB97-0540, and Comissionat per a Universitats i Recerca under Grant No. 1997SGR00090.
[1] M.C. Cross and P.C. Hohenberg, Rev. Mod. Phys. 65, 851 (1993).

[2] Chemical Waves and Patterns, edited by R. Kapral and K. Showalter (Kluwer Academic, Dordrecht, 1993).

[3] V.N. Biktashev, A.V. Holden, and H. Zhang, Philos. Trans. R. Soc. London, Ser. A 347, 611 (1994).

[4] A.V. Panfilov, J.P. Keener, Physica D 84, 545 (1995); A.T. Winfree, ibid. 84, 126 (1995).

[5] A.V. Panfilov and P. Hogeweg, Science 270, 1223 (1995).

[6] A.T. Winfree, Science 266, 1003 (1994); R.A. Gray, J. Jalife, A.V. Panfilov, W.T. Baxter, C. Cabo, J.M. Davidenko, and A.M. Pertsov, ibid. 270, 1222 (1995); A.T. Winfree, ibid. 270, 1224 (1995).

[7] F. Fenton and A. Karma, Chaos 8, 20 (1998); Phys. Rev. Lett. 81, 481 (1998).

[8] R. Gray and J. Jalife, Int. J. Bifurcation Chaos Appl. Sci. Eng. 6, 415 (1996).

[9] A.P. Muñuzuri, V. Pérez-Muñuzuri, and V. Pérez-Villar, Phys. Rev. E 58, 2689 (1998).

[10] M. Vinson, A. Pertsov and J. Jalife, Physica D 72, 119 (1993).

[11] I. Sendiña-Nadal, A.P. Muñuzuri, D. Vives, V. PérezMuñuzuri, J. Casademunt, L. Ramírez-Piscina, J.M. Sancho, and F. Sagués, Phys. Rev. Lett. 80, 5437 (1998).

[12] I. Sendiña-Nadal, S. Alonso, V. Pérez-Muñuzuri, M. GómezGesteira, V. Pérez-Villar, L. Ramírez-Piscina, J. Casademunt, J.M. Sancho, and F. Sagués, Phys. Rev. Lett. 84, 2734 (2000).

[13] P. Jung and G. Mayer-Kress, Phys. Rev. Lett. 74, 2134 (1995).

[14] S. Kàdàr, J. Wang, and K. Showalter, Nature (London) 391, 770 (1998).
[15] H. Hempel, L. Schymansky-Geier, and J. García-Ojalvo, Phys. Rev. Lett. 82, 3713 (1999).

[16] B.J. Welsh, J. Gomatam, and A.E. Burgess, Nature (London) 304, 611 (1983); A.T. Winfree and S.H. Strigatz, ibid. 311, 611 (1984); A.V. Panfilov, A.N. Rudenko, and V.I. Krinsky, Biophys. J. 31, 926 (1986); W. Jaknke, C. Henze, and A.T. Winfree, Nature (London) 336, 662 (1988); P.J. Nandapurkar and A.T. Winfree, Physica D 35, 277 (1989).

[17] C. Henze, E. Lugosi, and A.T. Winfree, Can. J. Phys. 68, 683 (1990); K.I. Agladze, R.A. Kocharyan, and V.I. Krinsky, Physica D 49, 1 (1991); H. Yamada and K. Nozaki, J. Phys. Soc. Jpn. 63, 379 (1994); T. Amemiya, P. Kettunen, S. Kàdàr, T. Yamaguchi, and K. Showalter, Chaos 8, 872 (1998).

[18] S. Mironov, M. Vinson, S. Mulvey, and A. Pertsov, J. Phys. Chem. 100, 1975 (1996); M. Vinson, S. Mironov, S. Mulvey, and A. Pertsov, Nature (London) 386, 477 (1997).

[19] M. Vinson and A. Pertsov, Phys. Rev. E 59, 2764 (1999).

[20] A.S. Mikhailov, Chaos Solitons Fractals 5, 673 (1995).

[21] A.S. Mikhailov, Foundations of Synergetics I (SpringerVerlag, Berlin, 1990).

[22] A.M. Pertsov, E.A. Ermakova, and A.V. Panfilov, Physica D 14, 117 (1984).

[23] E.A. Ermakova, A.M. Pertsov, and E.E. Shnol, Physica D 40, 185 (1989).

[24] J. García-Ojalvo and J. M. Sancho, Noise in Spatially Extended Systems (Springer-Verlag, New York, 1999).

[25] M. Ruiz-Villarreal, M. Gómez-Gesteira, C. Souto, A.P. Muñuzuri and V. Pérez-Villar, Phys. Rev. E 54, 2999 (1996).

[26] C. W. Gardiner, Handbook of Stochastic Methods, 2nd ed., Springer Series in Physics Vol. 13 (Springer, Berlin, 1989). 